\title{
VENOUS THROMBOSIS AND EMBOLISM
}

\author{
LESTER W. LOWE \\ From Northwick Park Hospital and Clinical Research Centre, Harrow
}

\begin{abstract}
After the operation of hip replacement arthroplasty, deep vein thrombosis in the legs and pelvis can be detected in 30 to 50 per cent of cases and pulmonary embolism in 10 per cent, carrying a mortality of one to two per cent. Thrombi can be found in 70 per cent of elderly patients operated on for hip fractures. Knee replacement fares no better and deep vein thrombosis has recently been found in 80 per cent (McKenna et al. 1980). No one can dispute these figures. However, the majority of orthopaedic surgeons would comment that, in the light of their own clinical experience, venous thrombosis is not such a serious problem, but would admit that the figure of mortality may be disguised by the passage of time in series that are unlikely to average more than 100 cases each year. The purpose of a review article is either to portray a condition which is too infrequent to command general expertise, or to survey a subject where controversy reigns. In the latter respect, the subject of venous thrombosis and embolism could not have been a better choice. Irrespective of whether the discussion is on points of diagnosis, clinical significance, prophylaxis or treatment, it is likely that any further facts and figures will draw an endless supply of contestants from the ranks of expert or amateur investigators alike. Perhaps the basic pathological changes in venous thrombosis are the only features which remain beyond contention. In a review of progress in orthopaedics during the past 25 years, Chalmers (1980) excludes any mention of this subject, and wisely so!

Pulmonary embolism and mortality were the initial stimuli to interest but the incidence was too small for the assessment of prophylactic measures. Death without warning had already demonstrated that the diagnosis of venous thrombosis using clinical signs was of very limited value. Ten years ago, new methods of detection using ${ }^{125}$ I-labelled fibrinogen, Doppler blood-flow recordings and phlebography became popular and demonstrated the high rate of occurrence with which we are now familiar. There was renewed interest in prophylaxis and for such a common condition it did not seem that an answer, which would at least improve the situation, would take long to find. Unfortunately, the large numbers of publications have only produced conflicting information and no single agent or method
\end{abstract}

has gained general acceptance. Furthermore, it is possible that these methods are too sensitive to venous thrombosis to provide an indicator of the potential clinical problem and insufficient effort has been put into separating the total incidence into significant and non-significant fractions. There is a risk that the tail is wagging the dog and the problem for the clinician is how to see perspective in the efforts of the investigators who may be guilty of placing too much emphasis on venous thrombosis as a convenient but unnatural end-point.

Research on the subject ranges from chemistry, cell biology and haematology, to radio-diagnosis and all aspects of conventional clinical care so that it is difficult to construct a review which is comprehensible to the orthopaedic surgeon and yet retains credence with the scientist. Some fundamental information is important for the understanding of this difficult subject and essential for the design of any future research into prophylaxis which might reduce the present confusion. Small surveys which test single agents are unlikely to be helpful. A reasoned multidisciplinary approach, leading to trials involving many centres, should be the framework for the future.

\section{DEVELOPMENT OF THROMBOSIS}

There are important differences between arterial and venous thrombosis.

In an artery injury to the endothelium of the wall exposes subintimal collagen, and the adhesion and aggregation of platelets to this area becomes the initial phase of "white" thrombus formation. Strands of fibrin with enmeshed erythrocytes and leucocytes are added and the proliferating clot streams in the direction of blood flow. In arterial thrombosis, therefore, the initiating features are vascular injury and platelet aggregation while a coagulation process (producing fibrin) plays a secondary part.

In a vein silted red cells, platelets and white cells in a valve pocket become bound together and anchored by fibrin to form a nidus. Successive layers of cells and fibrin are added in the direction of blood flow to produce a "red" thrombus, but when further propagation obstructs venous flow, retrograde thrombosis occurs. It is now considered that activation of the coagulation 
system is primarily responsible for the formation of the venous clot, and that the other features of platelet aggregation, stasis and endothelial damage play secondary, but nonetheless important, roles.

The blood is normally kept in a fluid state in the veins by a series of defence mechanisms. Intact venous endothelium is non-thrombogenic and it generates prostacyclin (which is an anti-platelet-aggregating agent) and a plasminogen activator (which lyses any intravascular fibrin). The blood itself contains "plasma inhibitors" which can neutralise any activated clotting factors whenever these are formed; of the six recognised inhibitors anti-thrombin III is the best known. The reticulo-endothelial system and the liver are responsible for clearing activated clotting factors and platelets from the circulation. Fibrinolysis, through the plasminogen system, effectively destroys any foci of fibrin. Intravascular clotting will therefore only proceed to recognisable levels when there has been a breakdown of some part of this defence mechanism. As an operation will upset the balance, the relative importance of hypercoagulability, platelet aggregation, stasis, endothelial damage and fibrinolysis should be examined because these would influence the control of intravascular clotting.

Hypercoagulability. Trauma or operation is followed by an "acute-phase reaction" which lasts for a period of 6 to 10 days and perhaps longer after the major soft-tissue dissection involved in operations on the hip or knee. There is an increase in factor VIII and fibrinogen levels and a slow fall of anti-thrombin III, but most important of all is the immediate activation of factor $X$ by the release of thromboplastins from damaged muscles and other tissues. This produces a state of hypercoagulability. Factor $\mathrm{X}$ is the focal point of intrinsic and extrinsic clotting pathways. In the presence of its activated form together with calcium and factor $\mathrm{V}$, prothrombin is converted to thrombin which then reacts with fibrinogen to produce fibrin.

Anti-thrombin III is one of the naturally occurring "plasma inhibitors" in the blood. It is a natural inhibitor of activated factor $X$ and therefore holds in check the hypercoagulable state which the presence of activated factor $\mathrm{X}$ confers. The importance of anti-thrombin III is threefold. First, it tends to be slowly reduced in amount by the "acute-phase reaction" after operation. Secondly, it is reduced in amount by oral contraceptive agents, perhaps by age and rarely for congenital reasons. Finally, its effect is considerably enhanced by a very low concentration of heparin; this is the whole basis of heparin prophylaxis.

Platelet aggregation. Platelet aggregation and adhesion to the damaged wall is the first feature of arterial thrombosis. However, Sevitt (1974) studied 50 recent thrombi in femoral veins and found that virtually all were lying over intact endothelium. Furthermore, the primary thrombi were either free of platelets or poor in platelets, confirming that platelets probably do not play an important role in the formation of the original nidus. Although it is important for the eventual evolution of the venous thrombosis, platelet aggregation must be seen as a secondary rather than an initiating factor. The number and stickiness of platelets are increased after operation but, in surgical patients screened by the ${ }^{125}$ I-labelled fibrinogen test, Flute et al. (1972) did not find any correlation between these changes and the incidence of deep vein thrombosis.

Stasis. In the presence of normal intima, retarded blood flow alone cannot initiate thrombosis and some alteration in the circulatory constituents of the blood must first be present. However, stasis is likely to have secondary effects on venous thrombosis. It increases the viscosity of blood and the tendency for cells to "silt" into the valve pockets. Also, the clearance of activated clotting factors by the liver and reticulo-endothelial system is reduced or prevented.

Endothelial damage. In the development of deep vein thrombosis, unlike that of arterial thrombosis, endothelial lesions alone are unlikely to be a major factor. The deposition of fibrin which is induced by local vascular injury is usually contained by normal compensatory mechanisms of fibrinolysis, but if these are overwhelmed by the presence of the hypercoagulable state thrombosis will proceed to clinically recognisable forms by the extension of deposits of platelets and cells within the fibrin network. This might explain why Stamatakis et al. (1977) found that after hip replacement the femoral vein was the site of the primary thrombus in 57 per cent of all cases of thrombosis. In contrast, two series involving general surgical patients demonstrated that the primary thrombus lay proximal to the calf in only 12 per cent (Nicolaides and O'Connell 1975) and 33 per cent (Browse and Thomas 1974) respectively.

Endothelial changes may not be confined to local veins. Trauma is thought to produce a systemic increase in vascular permeability which allows a migration of leucocytes across the endothelial sheets and accumulation between the endothelium and the basement membrane. Endothelial separation and desquamation follows and contributes to thrombosis, particularly in conditions of stasis and in the presence of the hypercoagulable state. This could explain a priming effect which promotes thrombosis in the contralateral limb.

Fibrinolysis. Fibrinolysis is an important part of the normal mechanism for keeping blood in its fluid state. Plasminogen activators in the vascular endothelium are released after venous occlusion, strenuous exercise and the use of vasoactive drugs. Price et al. (1980) have found that removal of a tourniquet after two and a half hours is followed for a period of an hour and three-quarters by an increase in fibrinolytic activity which continues at a very low level for a further 48 hours. Unfortunately, this is not seen to be of sufficient magnitude to be useful in a prophylactic sense. 


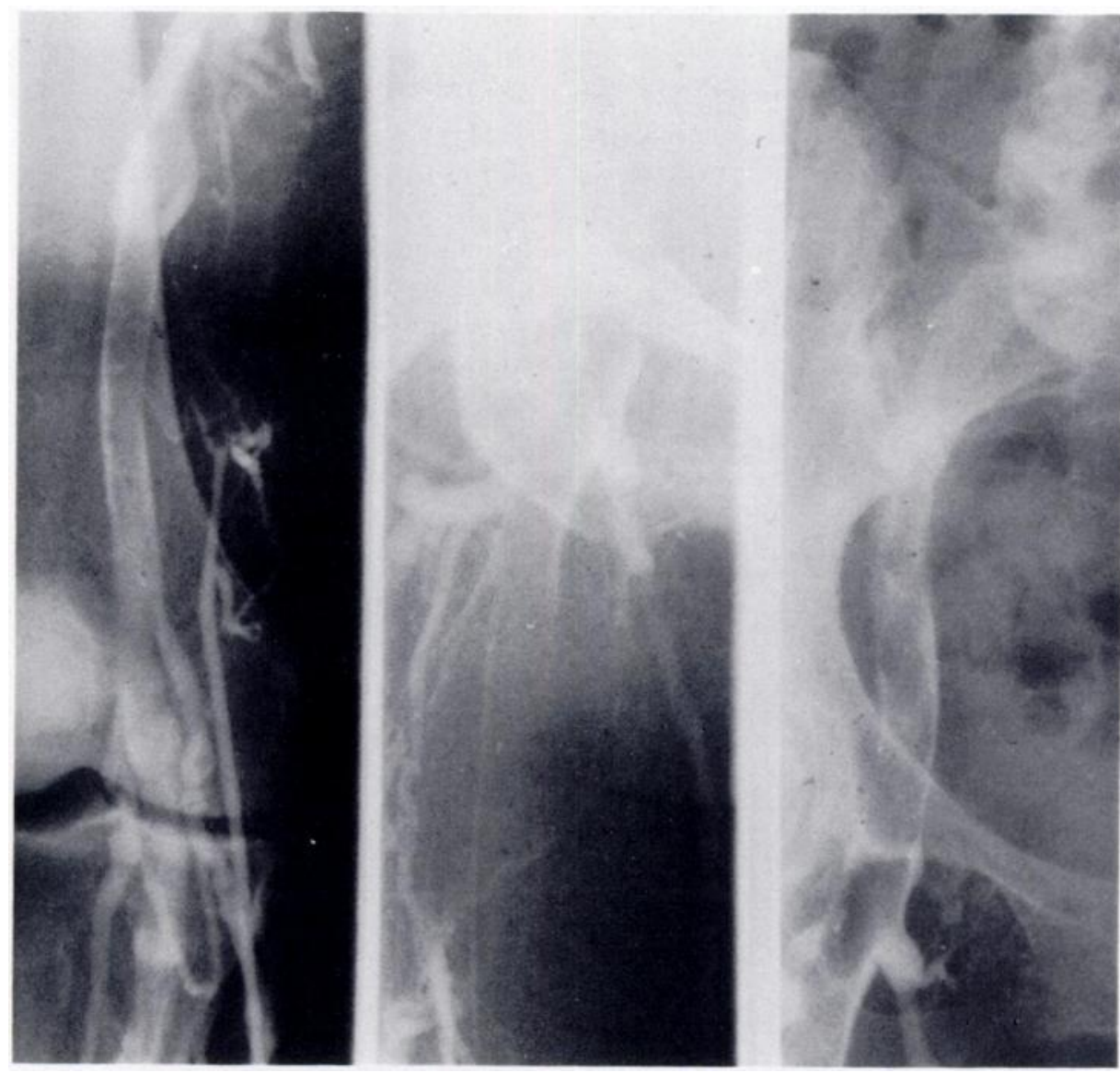

Fig. 1

Massive thrombosis extending from the popliteal vein to the proximal part of the external iliac vein.

However, venous thrombosis in the arms is relatively infrequent and it is interesting to note that plasminogen activity of the arm veins is three to four times higher than that in the legs (Nilsson 1974).

\section{DIAGNOSIS}

A diagnosis of pulmonary embolism is usually made on the history and signs of an abrupt respiratory incident coupled with an abnormality on lung scan. Many elderly patients will have pre-existing abnormalities in lung perfusion so that a single scan can only support rather than confirm a suggestion of pulmonary embolism.

The diagnosis of deep vein thrombosis is less satisfactory. Clinical criteria of swelling below the knee, calf pain with deep tenderness and a positive Homans' sign, together with contributory evidence of pyrexia, tachycardia, bluish discoloration of the skin and arterial spasm, produce an incidence of 10 per cent after hip operations (Crawford, Hillman and Charnley 1968). Unfortunately, this clinical diagnosis will be incorrect on 50 per cent of occasions and these signs will also fail to detect 50 to 90 per cent of thrombi in the deep venous system (Salzman and Harris 1976). Caution is needed in the interpretation of those series which use these criteria exclusively for the evaluation of prophylactic methods.
The reliability of clinical diagnosis seemed poor until Singer (1980) demonstrated a better correlation with clinical signs when the thrombus (identified by phlebography in his study) was situated in the larger proximal veins-which is where the risk lies.

Newer methods of investigation have been responsible for early and accurate diagnosis, for the identification of the patient at risk from embolism who may then be given full anticoagulant or thrombolytic treatment, and for more precise evaluation of different forms of prophylactic management. Unfortunately, each method has disadvantages which are made worse in orthopaedic surgery because of haematoma and soft-tissue swelling at the site of operation. The interpretation of the results is also made difficult by our ignorance of the incidence of venous thrombosis in comparable groups of medical patients or in the general population. Three tests are in common use.

The iodine-labelled fibrinogen test (Kakkar et al. 1970). Iodine-125 emits soft gamma radiation and incorporation into the clot can be detected by an external scintillation counter. A single injection of 100 microcuries (given one hour after operation to avoid too much loss into the wound) will allow daily scans for up to 10 days before a booster dose is required, and a daily check can be kept by a non-invasive procedure. If the counts 
are suspicious, phlebography should be performed to determine the exact location and size of the thrombus. Those proximal to the popliteal vein or greater than five centimetres in length in the calf veins are "significant" because of the immediate risk of embolism and the long-term possibility of venous hypertension (Figs 1 and 2 ). They should be treated by full anticoagulation with heparin and a period of five to seven days in bed to allow stabilisation of the thrombus. The remainder, clots of less than five centimetres in length in the calf veins (Fig. 3 ), are "not significant" and may be observed by continuing ${ }^{125} \mathrm{I}$ scanning. On the basis of this selection,
Ascending phlebography. This is the most complete and certain way of establishing the diagnosis but there are drawbacks. Phlebography is not exhaustive, it fails to display the profunda femoris vein and its tributaries in 40 per cent of cases and does not display all of the calf veins (Browse 1976). There is a risk of inducing thrombosis (perhaps as high as 15 per cent) or promoting its extension when already present because the sluggish circulation results in longer retention of the contrast medium. It is not repeatable and entirely unsuitable for use as a screening test. Its value lies in confirmation of a suspicious diagnosis and the demons-

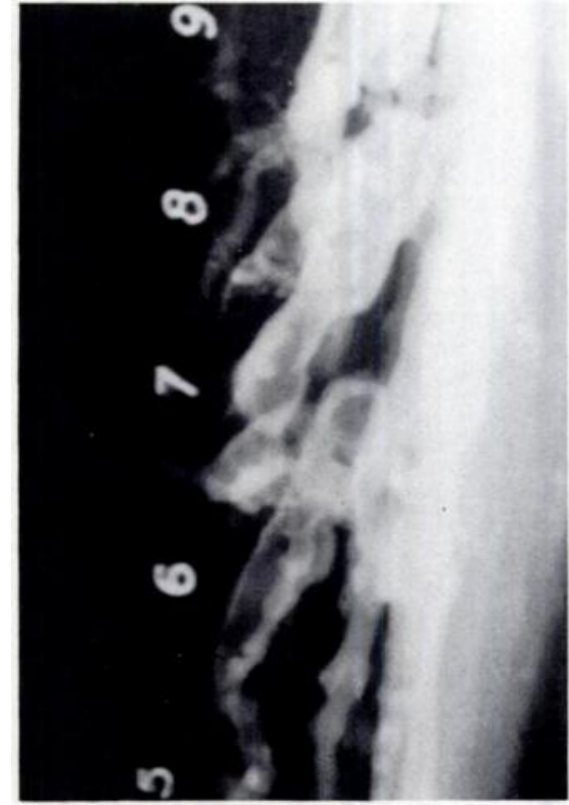

Fig. 2

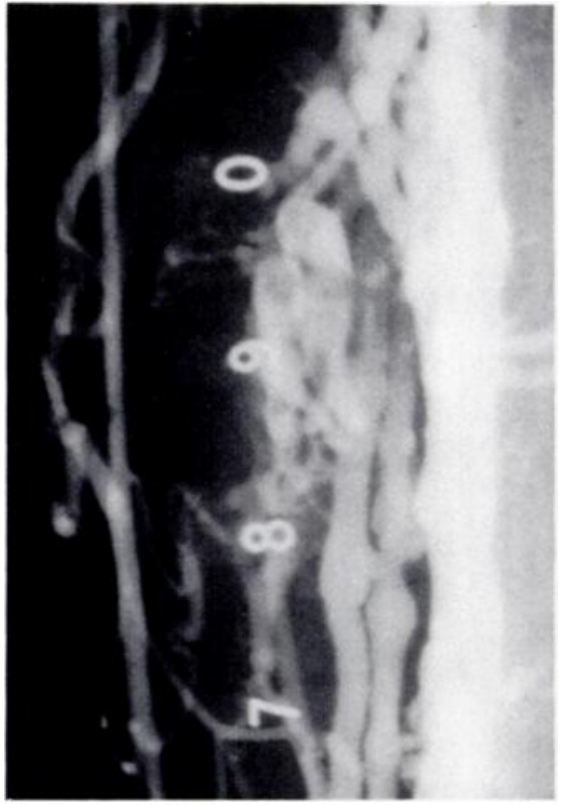

Fig. 3

Figure 2-Multiple thrombi exceeding five centimetres in length in the soleal plexus. Figure 3-Thrombi smaller than five centimetres in the soleal plexus.

an overall incidence of 42 per cent is reduced to 20 per cent for significant thrombosis requiring immediate treatment. These are arbitrary criteria but the policy can be justified because, once a minor thrombus has been recognised, it is rare for a patient to require anticoagulants at a later stage because of extension. Anticoagulation should not be started without good reason because it carries serious local risks for the orthopaedic patient as well as the need for long-term supervision of the dose.

Unfortunately, the ${ }^{125}$ I test cannot detect a thrombus proximal to the inguinal ligament or in the vicinity of a recent operation because of background activity. Not only are these dangerous areas but hip operations are known to be followed by a high incidence of primary thrombi in the ipsilateral femoral vein. It is unsatisfactory to rely solely on this test for screening purposes in orthopaedic surgery. Ideally, one of the ultrasound methods should also be used but considerable manpower and resources are necessary if all patients at risk are to be supervised in this way. tration of the position and extent of the thrombus so that therapeutic management can be decided. Mansfield (1977) has suggested that, if thrombolytic therapy is used, repeat phlebography is necessary to monitor its success.

The Doppler ultrasound flow detector. This method is most useful for the femoral and iliac veins and we have used it at Northwick Park Hospital to complement the deficiencies of the ${ }^{125}$ I-labelled fibrinogen test. It is, however, time-consuming and observer error is high.

${ }^{125}$ I-clearance value. This test has proved to be a useful by-product of the ${ }^{125}$ I-labelled fibrinogen test. The half-life reduction in radioactivity after injection normally exceeds 2.8 days. Increased utilisation of fibrinogen causes the half-life to fall, and in the absence of other conditions such as sepsis and bleeding, this has proved to be a sensitive indicator of venous thrombosis. A half-life of less than 2.8 days is suggestive of venous thrombosis and if less than 2.2 days the diagnosis is almost certain. Even more encouraging is its indication 
of a major or "significant" thrombosis which requires anticoagulation, since 87.5 per cent of these "significant" thromboses were associated with a half-life of less than 2.8 days compared with 77 per cent of all the thromboses detected (Lowe 1979). A half-life of less than 2.8 days should be taken as an indication for phlebography (in both legs if necessary) even if no warning comes from the ${ }^{125} \mathrm{I}$ scan or the Doppler flow recordings.

Laboratory diagnosis by changes in certain clotting factors. Heyse-Moore (personal communication) has found a close correlation between venous thrombosis after hip replacement and changes in plasma heparinneutralising activity, factor VIII:C and urinary $\beta$-thromboglobulin. At this stage, it is uncertain whether the alteration in levels occurs sufficiently early to be clinically useful in the diagnosis. Crandon et al. (1980) use a complicated formula involving euglobulin-lysis time, concentration of fibrin-related antigen, age and weight to offer a prediction on the risk of developing venous thrombosis after operation. This is important because of its implications for manpower and resources required for screening all patients.

MAVIS (mobile artery and vein imaging system). The equipment for ultrasonic angiography was developed from the principle of a continuous wave detector to assess Doppler shift in moving blood. An imaging concept was introduced by adding a storage monitor and a probe position resolver which produced spots on the

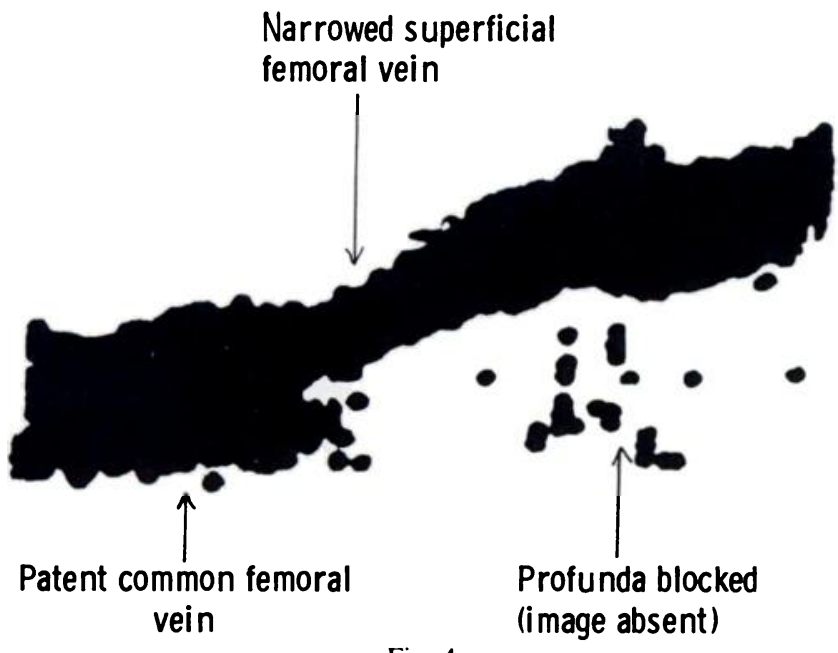

Fig. 4

Lateral view of the bifurcation of the femoral vein shown by Doppler angiography. There is a partially occluding thrombus in the superficial femoral vein and the profunda is completely occluded.

screen at different points as the probe was moved over the skin. The next advance was to use a pulsed system which allowed the operator to detect moving blood; thus the concept of imaging flow was introduced. This system worked well but the limitation was the length of time taken to build up the image of the vessel (or rather of the blood within it). MAVIS goes some way to overcoming this by using 30 detectors. It also has a direction- resolving device so that the blood moving in the artery and vein can be separated.

In practice there is a correlation of 92 per cent with phlebography for occluding thrombi in the popliteal and femoral veins, so that it is excellent for these difficult areas (Day, Fish and Kakkar 1976). One continuing disadvantage compared with radiography is that muscle is poorly penetrated, with loss of the signal strength, so that calf veins are less well displayed and give a correlation with phlebography of only 64 per cent. However, the method has considerable potential and preserves the important non-invasive feature which makes it suitable as a screening procedure (Fig. 4).

In summary, the ${ }^{125}$ I-labelled fibrinogen scan, the Doppler flow recordings and the ${ }^{125}$ I-clearance values offer the best combination for raising the suspicion of venous thrombosis, given adequate resources. Phlebography should then be used to identify the need for full anticoagulation. The best hopes for the future lie in identification of the patient at risk and the potential of ultrasound as a screening technique.

\section{PROPHYLAXIS}

Prophylaxis is the most controversial aspect of this subject and whereas a detailed review is impractical, it is pertinent to discuss some aspects of the more popular methods.

Rationale. On the basis of the aetiological evidence, venous thrombosis after operation should be most easily controlled hy the reduction or elimination of the hypercoagulable state. It is possible to do this at various stages in the clotting process but the most logical is the enhancement of a naturally occurring mechanism such as the ability of anti-thrombin III to neutralise activated factor X. Heparin has received much attention because this particular effect of anti-thrombin III is greatly increased by the presence of heparin at levels which are too low to influence the normal process of extravascular clotting required for surgical safety.

Platelet aggregation and stasis seem to be features of secondary importance so that, theoretically, aspirin, hydroxychloroquine, elastic stockings, pneumatic leggings and electrically induced calf stimulation are unlikely to be effective as single agents. The integrity of the venous endothelium, also of secondary importance in clot formation, remains largely beyond our control but methods to avoid desquamation (producing focal injury) and endothelial exhaustion (interrupting spontaneous fibrinolysis) may be important additions to future prophylaxis.

For the same fundamental reasons, it has been suggested that anticoagulants have little value in the prevention of thrombosis in coronary, cerebrovascular and peripheral arterial disease where the formation of the clot depends more on platelet aggregation and adhesion to damaged endothelium than the presence of 
a hypercoagulable state. Their benefit in these conditions is restricted to reducing complications such as embolism or further growth of thrombus.

\section{Small doses of subcutaneous heparin}

The actions of heparin are complicated and not fully understood but, depending on the plasma concentration, two main effects can be defined.

Anticoagulant effect (Fig. 5). This is produced by plasma levels of heparin in the range of 0.5 to 2.0 international

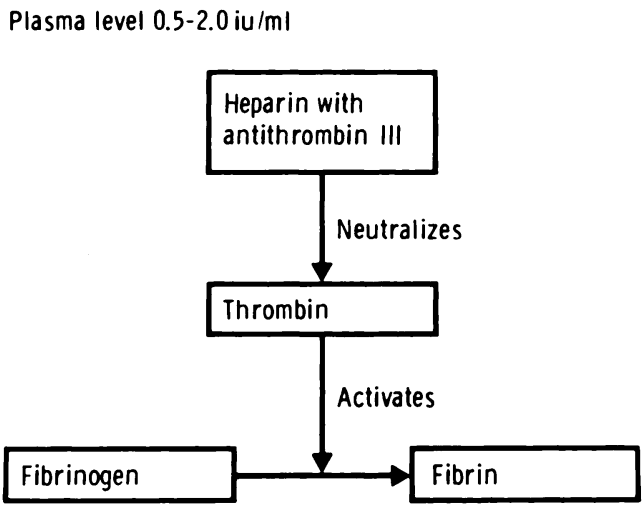

Fig. 5

The anticoagulant effect of heparin.

units per millilitre (iu/ml). Heparin forms a complex with anti-thrombin III which not only neutralises activated factor $\mathrm{X}$ but also neutralises thrombin before it can split fibrinopeptides $A$ and $B$ from fibrinogen. The clotting process is therefore halted. About 1000 micrograms of anti-thrombin III in the presence of this concentration of heparin will neutralise 1600 units of thrombin.

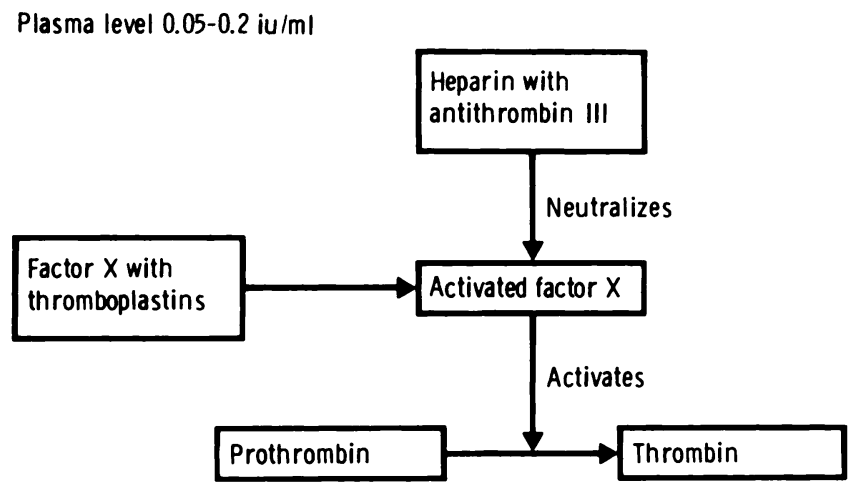

Fig. 6

The antithrombotic effect of heparin.

Antithrombotic effect (Fig. 6). The antithrombotic effect is achieved by plasma levels of heparin of 0.05 to 0.2 $\mathrm{iu} / \mathrm{ml}$, which are only one-tenth of the level required for anticoagulation (Lowe 1979). Heparin again forms a complex with anti-thrombin III which considerably enhances its power to neutralise activated factor $\mathbf{X}$.
Activated factor $\mathrm{X}$ is produced by the interaction of thromboplastins from tissue trauma at the time of operation with existing factor $\mathbf{X}$ in the circulating blood and it is an important feature of the hypercoagulable state. Given persistence in the blood, activated factor X potentiates the conversion of prothrombin to thrombin. This is a highly selective mechanism because one microgram of anti-thrombin III in the presence of 0.05 to $0.2 \mathrm{iu} / \mathrm{ml}$ of heparin in vitro will neutralise 32 units of activated factor $\mathrm{X}$ which, if allowed to persist, would generate 1600 units of thrombin. Heparin is usually given for this purpose by subcutaneous injection of 5000 iu every 8 or 12 hours in an attempt to achieve these levels by slow absorption. The haematological state remains "antithrombotic" (without any interference with normal haemostasis) as long as neutralisation of activated factor $X$ can exceed its generation.

Heparin in this low concentration of 0.05 to 0.02 $\mathrm{iu} / \mathrm{ml}$ also produces a significant decrease in blood viscosity (Erdi et al. 1976). At even lower levels produced by one international unit per kilogram it reduces platelet adhesiveness (Negus, Pinto and Slack 1971). Both features could contribute to the prophylactic effect.

There is general agreement about the value of subcutaneous heparin in controlling deep vein thrombosis and pulmonary embolism after elective abdominal and thoracic operations (Williams 1971; Kakkar et al. 1972,1975 ) and after myocardial infarction (Warlow $e t$ al. 1973). In contrast, its value after both acute and elective hip operations remains extremely controversial. For example, in the latter, Morris, Henry and Preston (1974) found a reduction in venous thrombosis from 50 per cent in the controls to 11 per cent in those on heparin, whereas Hampson et al. (1974) did not find any significant difference, with 54 per cent in the controls and 46 per cent in the treated group. The explanation for this discord lies in reasons which can be grouped as the variable clinical criteria in the design of the surveys; the widespread physiological effects of heparin; and the difficulty in maintaining the correct plasma levels of heparin.

Variable clinical criteria. The surveys varied in five respects.

Patient material. Postoperative thrombosis is probably less common in rheumatoid arthritis than it is in osteoarthritis. Morris et al. (1974) included more rheumatoid patients in the heparin-treated group and may have unfairly weighted their series whereas Hampson et al. (1974) excluded them.

Other drugs. Aspirin, indomethacin, phenylbutazone, ibuprofen and dextran all act synergistically with heparin. These drugs have not always been excluded or balanced between the control and treated groups.

Use of different methods of diagnosis. At the moment, this is an insoluble problem because any single method, or even combination of methods, has deficiencies. This is 
not obviated by the use of controls because some methods of prophylaxis may affect thrombosis in one part of the leg more than others.

Use of different preparations of heparin. Heparin is available as a calcium or sodium salt. Johnson et al. (1976) compared four preparations of heparin and found important differences in plasma levels after subcutaneous injection. The highest anti-factor $\mathrm{Xa}$ potentiating effect was obtained using sodium heparin of low molecular weight (mean 9000) with a mean level of $0.381 \mathrm{iu} / \mathrm{ml}$ three to four hours after the injection. Sodium heparin of high molecular weight (mean 15000) produced its highest level of $0.135 \mathrm{iu} / \mathrm{ml}$ at one hour. Calcium heparin gave much lower values $(0.072 \mathrm{iu} / \mathrm{ml}$ and $0.043 \mathrm{iu} / \mathrm{ml}$ for low and high molecular weights respectively). Sodium heparin is therefore preferred and calcium heparin is likely to give inadequate prophylactic levels. It is interesting that Morris et al. (1974) used sodium heparin and found it effective, in contrast to Hampson et al. (1974) who used calcium heparin.

Use of different operative approaches. This is not always stated and it is important because of variation in venous stasis, endothelial damage and the liberation of thromboplastins from damaged muscle. There is some evidence that the posterior approach to the hip is associated with a lower incidence of thrombosis than the lateral or anterior approaches (Lowe 1979).

The widespread physiological effects of heparin. Heparin has many different effects in the body which are beyond our control and this is a serious disadvantage in using it specifically as an antithrombotic agent. It forms complexes and binds with numerous proteins and cations, including collagen, insulin, histamine, vasopressin, lipoprotein, trypsin, adrenaline, anti-thrombin III, Christmas factor and platelet factor 4 . Some of these substances ca: compete for heparin more strongly than others. Anti-thrombin III has only a moderate affinity for heparin so that availability may be uncertain. One solution would be to combine heparin with antithrombin III in vitro before injection.

Difficulties in maintaining the correct plasma levels of heparin. At Northwick Park Hospital we started to use sodium heparin for prophylaxis after hip replacement six years ago with an empirical regime of 5000 iu eight-hourly. In a few early cases, heavy bleeding occurred at operation and we investigated the plasma heparin levels by the anti-factor Xa assay method (Denson and Bonnar 1973). Six elderly patients were given 5000 iu of sodium heparin and their resulting plasma levels are shown in Figure 7. Four of the patients showed levels which were in general agreement with those obtained by Yin, Wessler and Butler (1973) in young volunteers; one patient reached a peak level at three hours of $0.7 \mathrm{iu} / \mathrm{ml}$ which is well within the anticoagulant range; the remaining patient failed to show significant levels at any time after injection (all estimations lying along the abscissa) and was unpro- tected. These results demonstrated a serious disadvantage in using an empirical drug regime for elderly patients coming into hospital for elective operations on the hip; such a regime was perhaps a reason for the inconsistent results previously reported. They prompted a double-blind trial using a placebo and sodium heparin in doses which were individually tailored on the basis of a test dose response curve performed before operation.

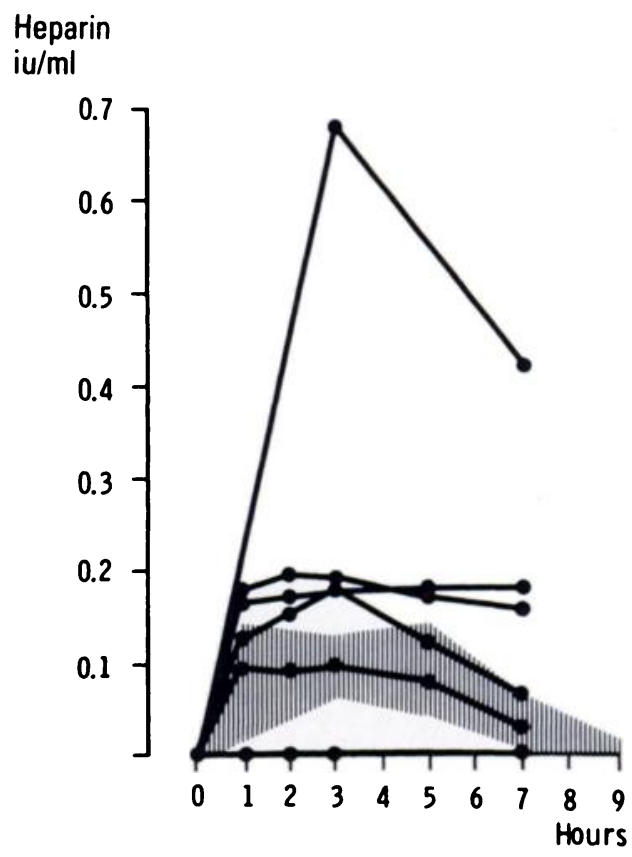

Fig. 7

Plasma heparin levels of six elderly patients following a single test dose of 5000 iu sodium heparin by subcutaneous injection. Shaded limits indicate the levels obtained by Yin $e t a l$. in young human volunteers.

The Northwick Park Hospital survey (Lowe 1979). We investigated the preoperative plasma heparin levels in 94 patients admitted for 100 hip replacement operations and from the results decided the postoperative dose and frequency of heparin for each individual patient. The patients were then randomly allocated to control and treated groups so that half received a placebo and half received sodium heparin for the whole of the postoperative stay in hospital (a minimum of 16 days). The code was retained by the pharmacy until the postoperative survey was complete. Deep vein thrombosis was confirmed by phlebography after detection by ${ }^{125} I-$ labelled fibrinogen scanning, Doppler blood-flow recordings and ${ }^{125}$ I-clearance values. Aspirin and other drugs likely to have an effect were discontinued 10 days before admission. The survey gave useful information on plasma heparin levels, on the incidence of deep vein thrombosis and pulmonary embolism using a tailored dose of heparin and on complications arising from this prophylactic method.

The plasma heparin levels in the preoperative 
investigation are shown in Figures 8 and 9. Two hours after the injection of 5000 iu, 71 patients ( 75 per cent) had levels within the band of 0.05 to $0.20 \mathrm{iu} / \mathrm{ml}$. In 11 patients ( 12 per cent) the levels were dangerously high, including one patient who was anticcagulated on the prophylactic effect. These figures mean that an empirical dose of 5000 iu of sodium heparin eight-hourly in this selection of patients would have given constant prophylactic protection to 52 per cent but 18 per cent could have reached anticoagulant levels. The remaining

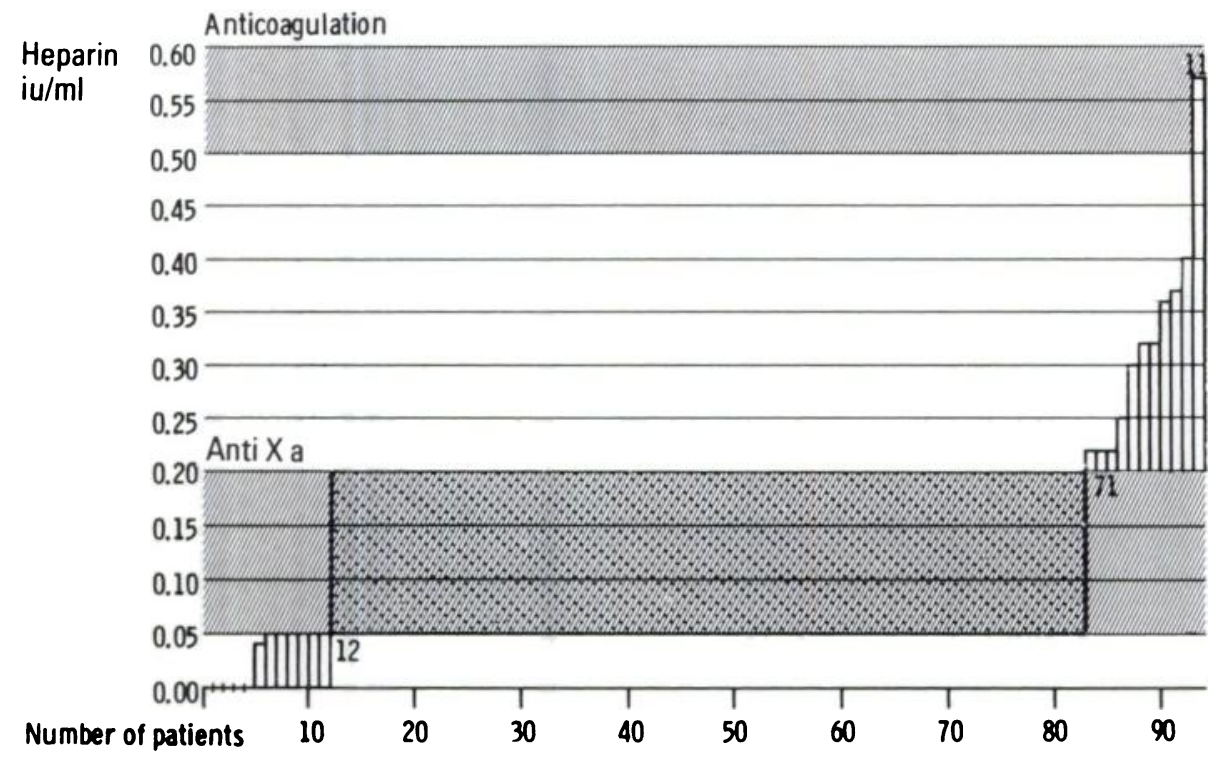

Fig. 8

Plasma heparin levels two hours after the injection of 5000 iu sodium heparin subcutaneously in 94 patients. Levels between 0.05 and $0.2 \mathrm{iu} / \mathrm{ml}$ should offer prophylaxis ( 71 patients). Levels above 0.20 $\mathrm{iu} / \mathrm{ml}$ may produce an anticoagulant effect with single or subsequent injection ( 11 patients). Levels less than $0.05 \mathrm{iu} / \mathrm{ml}$ are probably ineffective (12 patients).

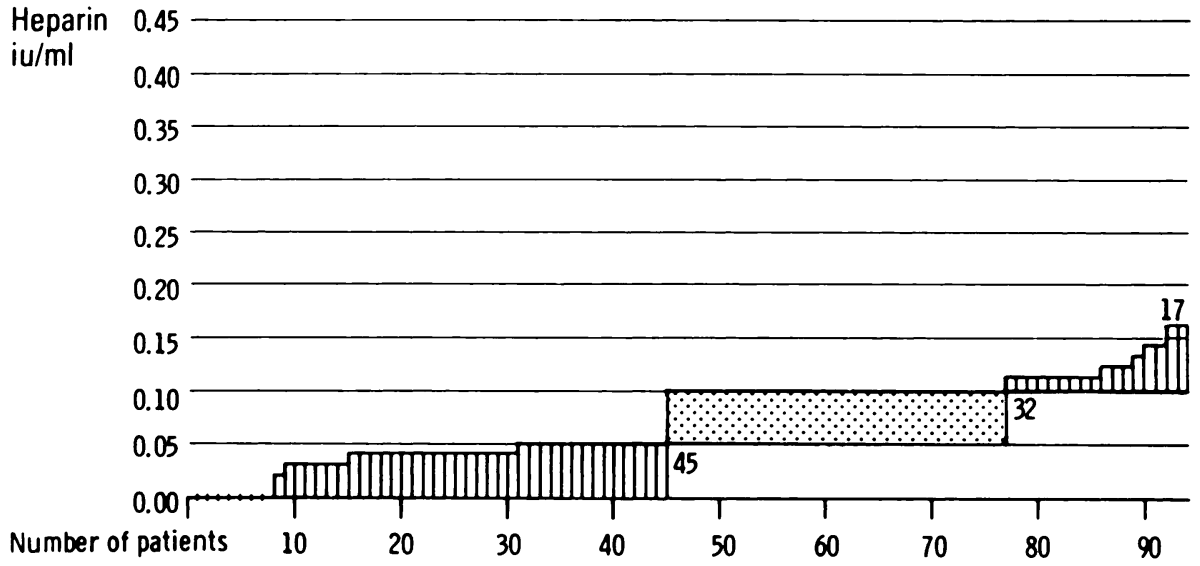

Fig. 9

Plasma heparin levels eight hours after the injection of 5000 iu sodium heparin subcutaneously in 94 patients. Levels between 0.05 and $0.10 \mathrm{iu} / \mathrm{ml}$ should offer safe prophylaxis (32 patients). Levels greater than $0.1 \mathrm{iu} / \mathrm{ml}$ may produce an anticoagulant effect with subsequent injections (17 patients). Levels less than $0.05 \mathrm{iu} / \mathrm{ml}$ are probably ineffective (45 patients).

single dose of $5000 \mathrm{iu}$. Twelve patients (13 per cent) had levels which would have been too low for a prophylactic effect. Eight hours after injection, 49 patients $(52$ per cent) had levels within the "prophylactic" band; however, about one-third of these (17 patients) had levels above $0.10 \mathrm{iu} / \mathrm{ml}$ so that anticoagulation levels would have been within reach from the summation effect of consecutive injections. Forty-five patients (48 per cent) had levels which would have been too low for a
48 per cent were either completely unprotected or only protected for a short time after each injection.

When the code for postoperative treatment was broken at the end of the survey, the 51 patients allocated to heparin had received the following regime: 15 had had 5000 iu 8-hourly, 32 had had 5000 iu 12-hourly, 1 had had 3000 iu 8 -hourly and 3 patients had had 3000 iu 12-hourly. In retrospect, some of these levels were too low and erred too much on the side of operative safety so 
that the prophylactic effect was not maximised. At the same time, not a single patient given heparin showed any sign of abnormal bleeding during or after operation.

Thrombosis occurred in 33 per cent of the controls and 14 per cent of the treated group (a statistically significant difference, $P<0.05$ ). If "significant" thrombosis is specifically considered, it occurred in 20 per cent of the controls and 12 per cent of the treated groups but this difference is not statistically significant. Thrombosis occurred, on average, 8.2 days after operation in the controls and 7.8 in the treated group so that there is no support for the contention by Hampson et al. (1974) that heparin does not reduce but only defers the development of thrombosis. The distribution of thrombosis was the ipsilateral leg in 75 per cent, the contralateral leg in five per cent and both legs in 20 per cent. Since this survey was completed, a further 131 hip replacement arthroplasties have been performed using individually tailored doses of sodium heparin. There were 11 cases (8.5 per cent) of "significant" thrombosis requiring full anticoagulation.

Pulmonary embolism occurred in 10 per cent of the controls and four per cent of the treated group but there were no deaths.

This survey suggests a definite place for subcutaneous heparin when the dose can be tailored to the patient. It is possible that the prophylactic effect could be increased by a more generous dose for patients with very low plasma levels without incurring any risks. If resources are not available for the estimation of plasma heparin levels by the anti-factor Xa assay, I consider that the empirical use of 5000 iu sodium heparin eighthourly should not be used in elderly patients because of the potential danger of reaching anticoagulant levels in 18 per cent of patients. If 5000 iu sodium heparin twice a day is used, this risk is reduced but not removed and prophylaxis will be less effective.

\section{Ultra-low doses of intravenous heparin}

In a double-blind randomised trial of 95 surgical patients, Negus et al. (1980) claim to have reduced the incidence of deep vein thrombosis from 22 per cent to four per cent by using ultra-low doses of intravenous heparin. To the saline drip which is normally used for three to five days in the general management of these cases they added 1 iu of sodium heparin per kilogram of body weight per hour, which represents a very small daily dose of about 1680 iu in the average sized patient and might be expected to give a plasma heparin level of only $0.007 \mathrm{iu} / \mathrm{ml}$. It is difficult to believe that the ultra-low dose can achieve an effect which has not been consistently seen from the higher plasma levels of small doses of heparin unless the route of administration is critical or heparin acts highly selectively at different concentrations. Further study is required and the results from its application in orthopaedic surgery will be awaited with interest.
There has been much discussion about the mechanism of action and it is unlikely to be due to a direct effect on the clotting process as we normally understand it. Perhaps the heparin induces release of lipoprotein lipase which reduces platelet stickiness and the tendency to form the platelet-fibrin nidus of venous thrombosis. A trigger effect releasing endogenous glycosaminoglycans which have an antithrombotic effect has been suggested. The heparin could liberate platelet factor 4 from the vascular endothelium because this happens at higher concentrations and possibly with much lower ones also.

\section{Intrapulmonary heparin}

Ultra-low dosage of heparin raises the question of the potential value of heparin administered by the pulmonary route. Jacques, Mahadoo and Kavanagh (1976) described a sustained concentration of heparin in the blood after inhalation of a single dose: this lasted for three days in dogs and 14 days in man. Inhalation was not followed by any sudden increase in plasma heparin levels so that it was suggested that heparin was rapidly cleared from the lung by the macrophage system and then slowly released into the plasma. In human volunteers, these workers used a single dose exceeding $1300 \mathrm{iu} / \mathrm{kg}$ administered by ultrasonic nebuliser and produced recognisable heparin levels in the plasma measured by anti-factor $\mathrm{Xa}$ assay. There was a linear relationship between the total response (percentage increase in clotting time multiplied by the duration in days) and the dose.

We have repeated this work at Northwick Park Hospital using nine human volunteers and single doses varying between 600 and $5000 \mathrm{iu} / \mathrm{kg}$. In five, the plasma heparin levels were less than $0.05 \mathrm{iu} / \mathrm{ml}$ at all times, three produced occasional levels between 0.06 and $0.07 \mathrm{iu} / \mathrm{ml}$ and one volunteer reached $1.5 \mathrm{iu} / \mathrm{ml}$ four hours after inhalation but the level fell to nothing after that.

Our inability to confirm the results of Jacques et al. is disappointing if we have the potentiation of antithrombin III in mind, but the method could have a place in prophylaxis through the mechanisms discussed for ultra-low doses of intravenous heparin. A clinical trial would be worthwhile at this stage and different types of nebuliser should be tested because droplet size is critical if the heparin is to reach the alveoli and not stop at the level of the bronchioles where presumably it would be ineffective.

\section{Aspirin}

Aspirin has an effect on platelets which, in theory, could be used for antithrombotic purposes even though platelet aggregation is not seen as the feature of primary importance in the initiation of venous thrombosis. Many of the clinical series involving aspirin have been blighted by the problems which seem common to the subject as a whole - small numbers of patients, lack of controls and defects of diagnosis. The literature generally reflects a 
low level of enthusiasm for its prophylactic effect in deep vein thrombosis with most of the pessimism coming from the orthopaedic investigators. The trial which was sponsored by the Medical Research Council (Butterfield et al. 1972) did not show any advantage from aspirin.

A small dose of aspirin $(3.4 \mathrm{mg} / \mathrm{kg})$ inhibits platelet aggregation through the enzyme prostaglandin cyclooxygenase. However, a slightly larger dose $(4.9 \mathrm{mg} / \mathrm{kg})$ will also inhibit the release of prostacyclin from the endothelium of the vessel wall (O'Brien 1980). Prostacyclin has already been mentioned as one of the agents responsible for keeping intravascular blood in its fluid state by acting as an anti-platelet-aggregating agent. Identification of the critical strength of aspirin that differentially inhibits some platelet functions without interfering with this prostacyclin production will therefore determine the efficacy of aspirin in preventing thrombosis. Considerable differences between individuals have been found in the response to aspirin which bring to mind the problems of maintaining plasma heparin levels for anti-thrombin III reinforcement, so that the use of aspirin in practice is not going to be easy, even when the theoretical requirements have been worked out.

\section{Dextran}

Dextrans are polysaccharides built up of glucose molecules. Dextran 70 (mean molecular weight 70000 ) has been shown to expand the blood volume for three to five days and is usually preferred to dextran 40 , which has a shorter effect and has been implicated as a cause of renal failure by increasing urinary viscosity (Bonnar 1977). Initially, it was felt that dextran might have an antithrombotic effect by reducing blood viscosity and improving blood flow. The viscosity of the blood in the veins is relatively higher than in arteries because the blood flow and shear rate are lower. Furthermore, Dormandy and Edelman (1973) found that patients who developed deep vein thrombosis had viscosities before operation which were 21 per cent higher on average than those who did not. This may be another facet of the identification of the patient at risk.

The action of dextran is probably more complicated than a simple effect on viscosity. It is a heparinoid which is effective in accelerating the inhibition of activated factor X by anti-thrombin III although it is 40 to 50 times less effective than heparin in this respect. It reduces platelet aggregation, alters the stability of fibrin so as to diminish the rate of growth of the thrombus and reduces the rise of the levels of factor V and factor VIII after operation.

The evidence in favour of using dextran as a prophylactic agent produces cautious optimism, its use is less controversial than heparin or aspirin but it has not received the same detailed attention. Berqvist $e t$ al. (1979) found that it was better than heparin for reducing deep vein thrombosis after fractures of the hip but neither dextran or heparin were effective after elective hip operations. A question on the validity of the series arises, however, because control is usually thought to be easier after elective hip operations than after fractures of the femoral neck. These workers used 500 millilitres during operation, immediately after operation and on the first and third postoperative days. Doubt still exists about the minimal effective dose and the amount required for a sustained antithrombotic effect (if any). There are no serious disadvantages to dextran except the need for intravenous injection in the postoperative phase, the cost and the very rare problems of hepatitis and renal failure.

Dextran does not produce increased bleeding. However, it has a synergistic effect with other antithrombotic agents which may increase their joint prophylactic effect but also produce a substantial risk from bleeding. Salvati and Lachiewicz (1976) combined dextran with aspirin or warfarin and had wound complications of 15 per cent and 24 per cent respectively. Their incidence of thrombo-embolic complications was less than 10 per cent but the diagnosis was made solely on clinical signs.

Janvrin (personal communication) has produced an interesting new twist to the situation using the Biobridge to measure whole blood coagulation by impedance clotting time. Anaemia and haemodilution with saline produce a hypercoagulable state, polycythaemia a hypocoagulable state, while haemodilution with dextran in saline produces no change. He argues that dextran prevents deep vein thrombosis not so much by an intrinsic action but more by preventing the hypercoagulable state which the infusion of saline alone would produce. In other words, he feels that the prevalence of thrombo-embolism is related to the popular use of intravenous saline at the time of operation. These ideas have been tested in 60 patients undergoing routine laparotomy. They were randomly allocated to receive intravenous fluid ("wet" group) or no intravenous fluid ("dry" group). The wet patients were significantly more hypercoagulable at the end of operation and 30 per cent developed deep vein thrombosis compared to seven per cent in the dry group $(P<0.05)$.

These findings are contrary to expectation based on blood viscosity. One possible explanation is that when blood is diluted with saline, those features striving to retain the fluid state lose their power to a greater degree than the wide range of participants involved in the instigation of the clotting process. It is an intriguing prospect to consider that a large portion of the thrombo-embolic problem could be iatrogenic.

\section{Other agents}

Warfarin and phenindione have been used for prophylaxis as true anticoagulants aiming for a prothrombin activity of 10 to 25 per cent (Crawford $e t$ al. 1968 ) or 5 to 15 per cent (Pinto 1970). Neither of these 
two series showed significant diminution in the incidence of thrombosis and the first was associated with so many bleeding problems that enthusiasm quickly faded and has not been revived.

Intravenous lignocaine is said to act on the white cells by limiting their adhesion to the endothelium and their invasion of the vessel wall. The effect should therefore be useful in conditions where stasis and endothelial damage have occurred. Cooke et al. (1977) reduced thrombosis from 78 per cent to 14 per cent in a small series of 14 patients during the first six days after operation but the incidence then climbed to 53 per cent by the fourteenth day.

Hydroxychloroquine (Plaquenil) inhibits platelet aggregation and adhesiveness. Its value is seen in a similar light to aspirin.

\section{Physical methods}

If venous slowing or stasis is important in thrombosis, physical methods to improve flow should have a place in prophylaxis. However, there is no general agreement that a significant proportion of venous thrombi start to form during the operation and it is more likely that origin and growth to a recognisable size occurs near the seventh day after operation. This reflects the importance of the hypercoagulable state rather than venous stasis because, by this time, most patients are mobile. Putting aside the theory, the obvious limitations of intermittent compression cuffs, electrical calf stimulation or elastic stockings are that the deep pelvic veins are beyond influence.

It is well recognised that a tubular stocking is counter-productive because the wedge shape of the leg produces differential pressures which are the reverse of what is required. However, correctly graduated stockings will produce a decrease in venous pressure at the ankle when walking and an increase in the rate of venous flow. A range of graduated stockings may not be adequate if there is unusual disproportion in the girth of the calf and ankle.

In reviewing these physical methods, Shaw (1977) found that an increase in blood flow was not enough so that even graduated stockings were ineffective. Only those methods which had a pulsatile element (foot pedals, compression cuffs, electrical stimulation) seemed to have any potential and a reduction of thrombosis from about 20 per cent in the controls to eight per cent in the treated groups was noted. He emphasised that there was no reason to hope that these forms of prophylaxis would decrease death from pulmonary embolism.

\section{THE PILL}

Anti-thrombin III activity drops precipitously with oral contraceptives. Von Kaulla et al. (1971) have expressed anti-thrombin III activity as anti-thrombin III time by exposing the serum to be treated to a known quantity of thrombin and then estimating the amount of thrombin remaining after six minutes' incubation. The shorter the time, the less anti-thrombin III activity is present. Normal women who were not on the Pill had an average time of 54.2 seconds. After 10 contraceptive pills the time fell to an average of 23.3 seconds, and after 20 , to an average of 19 seconds. In one woman who then discontinued the Pill, the anti-thrombin III time started to rise after nine days and had returned to normal after 18 days. Loss of anti-thrombin III activity of this degree does not necessarily imply subsequent formation of intravascular clots but it confers a propensity which may be realised by certain events such as vascular damage, stasis or hypercoagulability after operation.

The long-term effects of the Pill have been described by Sagar et al. (1976). After a mean duration of 34 months, anti-thrombin III activity was $76.3 \pm 2.8$ per cent compared with $91.6 \pm 2.8$ per cent in the controls who were not on the Pill. Any patient with a pre-operative value of less than 80 per cent should be regarded as carrying a five to six-fold higher risk of thrombosis. No relationship has been established between the type of oral contraceptive and the level of anti-thrombin III activity or the increase of venous thrombosis.

Heparin potentiates the effect of anti-thrombin III and reduces a further fall which occurs with surgical treatment but this does not mean that protection of the patient is necessarily raised to normal levels.

Agreement has not been reached on the policy to adopt. The functionally active anti-thrombin III can be measured by the chromogenic substrate method before admission to hospital. Depending on the apparent risk, one option is to discontinue the Pill for a period of time (so far unspecified but four weeks may be adequate) with the possible result of unwanted pregnancy. The alternative is to use small doses of subcutaneous heparin to reduce the risk as far as possible.

\section{TREATMENT}

Approximately a quarter of a million people in the United Kingdom suffer from the post-thrombotic syndrome. Thrombosis between the origin of the common femoral vein and the termination of the common iliac vein produces pulmonary embolism in $\mathbf{5 0}$ per cent of patients, in one-third of whom it is fatal; nearly every surviving patient develops the postthrombotic syndrome. O'Donnell et al. (1977) have followed the progress of 21 of these patients for 10 years and found ulcers of the leg in 18,14 of whom needed admission to hospital on five or more occasions and three required below-knee amputation. At a guess, the treatment of this sort of patient costs $£ 25000$ over 10 years. After deep vein thrombosis distal to the popliteal vein, about nine per cent suffer from a post-thrombotic syndrome (Bieger et al. 1976). 
Thrombosis is followed by a natural process of fibrinolysis. This is mildly enhanced by anticoagulation with heparin which also limits extension of the clot. Streptokinase indirectly activates plasminogen into plasmin and produces vigorous thrombolysis. However, once intravascular thrombosis is apparent, considerable damage may have occurred and if removal of the thrombus is not followed by repair, there could be no clinical benefit. There is a case for leaving the thrombus in place so that an alternative collateral circulation can develop.

Which, if any, patients with thrombo-embolism would benefit from thrombolytic treatment as opposed to anticoagulation alone? Sharp (1977) has reviewed the situation and could not find a series large enough to show that lysis of venous thrombi had lowered the incidence of pulmonary embolism and its mortality or that post-thrombotic morbidity in the leg was reduced. Lysis of pulmonary emboli produced a more rapid return of function and well-being in the first 72 hours but there was no difference compared with heparin after five days had passed. It was doubtful whether successful thrombolysis decreased the mortality of pulmonary embolism to a significant degree, and if the patient survived long enough for successful lysis to occur he would probably have survived anyway. One important advantage is that removal of one clot by thrombolysis offers a better chance of survival if repeated emboli occur.

The solution is uncertain but thrombosis proximal to the knee, common after hip operations, merits the consideration of thrombolysis with streptokinase. Very early diagnosis, with lysis, could have a better effect on embolism and the post-thrombotic syndrome than has yet been described.

\section{DISCUSSION}

No method of prophylaxis can be relied upon to remove the fear of deep vein thrombosis and its consequences, yet it is probably unwise to anticoagulate a postoperative orthopaedic patient until the diagnosis of a significant thrombus has been established with some certainty. The clear duty of the orthopaedic surgeon therefore is consistently to apply methods of diagnosis (other than clinical signs) to all patients who have had major operations on the hip or knee, particularly the elderly. This produces considerable reassurance for nurses, physiotherapists and junior medical staff for whom day-to-day problems such as pain and swelling carry constant foreboding because of the frequency of thrombo-embolism. Whether or not they are able to carry out this duty depends on technical equipment and manpower resources.

Morris (1980) asked 752 orthopaedic surgeons and 663 general surgeons what prophylactic methods, if any, they used with operations for fractures of the hip in the elderly, replacement arthroplasty of the hip and major abdominal and thoracic procedures. The results suggested that prophylaxis is inversely related to the frequency with which thrombo-embolism occurs. The enthusiasm of the general surgeons in their own field of the abdomen and thorax is probably due to confidence with low doses of heparin and its infrequent side-effects in their hands. The reverse is true for the orthopaedic surgeon for whom no easy effective and low-risk method of prophylaxis has emerged. In the case of the elderly patient with the fractured hip, there is the added difficulty that thrombosis may be already present in a high percentage when they are admitted to hospital and, for many, prophylaxis ought to be replaced by immediate anticoagulation which is inconsistent with early operative treatment.

Despite this, the orthopaedic surgeon might consider that attempts at routine prophylaxis should be tried. There is a choice between aspirin, dextran, ultra-low doses of heparin, hydroxychloroquine and pulsatile physical methods, all of which are reasonably safe but none effective with certainty. A low fixed dose of heparin is not recommended but the method may be valuable if individual prescription can be determined. The female patient on the Pill poses special problems.

Throughout this paper, future trends and developments have been discussed. Most optimism lies with ultrasound for diagnosis and the identification of the patient at risk. The latter would at least allow time and talent to be devoted to those who need it most.

\section{REFERENCES}

Berqvist D, Efsing HO, Hallböök T, Hedlund T. Thromboembolism after elective and post-traumatic hip surgery: a controlled prophylactic trial with dextran 70 and low-dose heparin. Acta (hir Scand 1979;145:213-8.

Bieger R, Boekhout-Mussert RJ, Hohmann F, Loeliger EA. Is streptokinase useful in the treatment of deep vein thrombosis? Acta Med Scand 1976:199:81-8.

Bonnar J. The prophylaxis of deep-vein thrombosis using dextran. In: Forbes $\mathrm{CD}$. Mackay N, eds. The detection and treatment of thrombo-('mbolism. A symposium held at the Royal College of Physicians and Surgeons, Glasgow, April 1976. Ashtead. Surrey: The Trust for Education and Research in Therapeutics, 1977:129-36.

Browse NL. Venous thrombo-embolism. Das Medizinische Prisma. Ingelheim am Rhein, West Germany: CH Boehringer Sohn, $1976 ; 2: 76$.

Browse NL, Thomas ML. Source of non-lethal pulmonary emboli. Lancet 1974;i:258-9.

Butterfield WJH, Hicks BH, Ambler AR et al. Effect of aspirin on postoperative venous thrombosis. Report of the Steering Committee of a trial sponsored by the Medical Research Council. Lancet 1972;ii:441-5.

Chalmers J. Orthopaedics during the past 25 years. J $R$ Coll Surg Edinb 1980;25:345-53.

Cooke ED, Bowcock SA, Lloyd MJ, Pilcher MF. Intravenous lignocaine in prevention of deep venous thrombosis after elective hip surgery. Lancet 1977;ii:797-9. 
Crandon AJ, Peel KR, Anderson JA, Thompson V, McNicol GP. Prophylaxis of postoperative deep vein thrombosis: selective use of low-dose heparin in high-risk patients. Br Med J 1980;281:345-7.

Crawford WJ, Hillman F, Charnley J. A clinical trial of prophylactic anticoagulant therapy in elective hip surgery. Centre for hip surgery, Wrightington Hospital, 1968; Internal publication 14.

Day TK, Fish PJ, Kakkar VV. Detection of deep vein thrombosis by Doppler angiography. Br Med J 1976;i:618-20.

Denson KW, Bonnar J. The measurement of heparin. A method based on the potentiation of anti-factor Xa. Thromb Diath Haemorrh 1973;30:471-9.

Dormandy JA, Edelman JB. High blood viscosity: an aetiological factor in venous thrombosis. Br J Surg 1973;60:187-90.

Erdi A, Kakkar VV, Thomas DP, Lane DA. Effect of low-dose subcutaneous heparin on whole-blood viscosity. Lancet 1976;ii:342-4.

Flute PT, Kakkar VV, Renney JTG, Nicolaides AN. The blood and venous thromboembolism. In: Kakkar VV, Jouhar AJ, eds. Thromboembolism: diagnosis and treatment: Edinburgh and London: Churchill Livingstone, 1972:2-12.

Hampson WGJ, Harris FC, Lucas HK et al. Failure of low-dose heparin to prevent deep-vein thrombosis after hip-replacement arthroplasty. Lancet 1974;ii:795-7.

Jacques LB, Mahadoo J, Kavanagh LW. Intrapulmonary heparin. Lancet 1976;ii:1157-61.

Johnson EA, Kirkwood TBL, Stirling Y et al. Four heparin preparations: anti-Xa potentiating effect of heparin after subcutaneous injection. Thromb Haemostas 1976;35:586-91.

Kakkar VV, Corrigan TP, Fossard DP et al. Prevention of fatal postoperative pulmonary embolism by low doses of heparin. International Multicentre Trial. Lancet 1975 ;ii:45-51.

Kakkar VV, Corrigan T, Spindler J et al. Efficacy of low doses of heparin in prevention of deep-vein thrombosis after major surgery. A double-blind, randomised trial. Lancet 1972;ii:101-6.

Kakkar VV, Nicolaides AN, Renney JTG, Friend JR, Clarke MB. ${ }^{125}$ I-labelled fibrinogen test adapted for routine screening for deep-vein thrombosis. Lancet 1970; i:540-2.

Lowe LW. The role of anticoagulants in hip surgery. In: McKibbin B, ed. Recent advances in orthopaedics. 3. Edinburgh, London and New York: Churchill Livingstone, 1979:31-55.

McKenna R, Galante J, Bachmann F, Wallace DL, Kaushal SP, Meredith P. Prevention of venous thromboembolism after total knee replacement by high-dose aspirin or intermittent calf and thigh compression. Br Med J 1980;280:514-7.

Mansfield AO. Detection of venous occlusion: venography. In: Forbes CD. Mackay N, eds. The detection and treatment of thrombo-embolism. A symposium held at the Royal College of Physicians and Surgeons, Glasgow, April 1976. Ashtead, Surrey: The Trust for Education and Research in Therapeutics, 1977:111-3.

Morris GK. Prevention of venous thromboembolism. Lancet 1980;ii:572-4.

Morris GK, Henry APJ, Preston BJ. Prevention of deep-vein thrombosis by low-dose heparin in patients undergoing total hip replacement. Lancet 1974;ii: $797-800$

Negus D, Cox SJ, Friedgood A, Peel ALG, Wells BW. Ultra-low dose intravenous heparin in the prevention of postoperative deep-vein thrombosis. Lancet 1980;i:891-4.

Negus D, Pinto DJ, Slack WW. Effect of small doses of heparin on platelet adhesiveness and lipoprotein-lipase activity before and after surgery. Lancet $1971 ; \mathbf{i}: 1202-5$.

Nicolaides AN, O'Connell JD. Origin and distribution of thrombi in patients presenting with clinical deep venous thrombosis. In: Nicolaides AN, ed. Thromboembolism: aetiology, advances in prevention and management. Lancaster: Medical and Technical Publishing Co. Ltd, 1975:167-80.

Nilsson IM. Haemorrhagic and thrombotic diseases. London: John Wiley, 1974:170-7.

O'Brien JR. Platelets and the vessel wall: how much aspirin? Lancet 1980;i:372-3.

O'Donnell TF, Browse NL, Burnand KG. Thomas ML. The socioeconomic effects of an iliofemoral venous thrombosis. $J$ Surg Res 1977;22:483-8.

Pinto DJ. Controlled trial of an anticoagulant (warfarin sodium) in the prevention of venous thrombosis following hip surgery. Br $J$ Surg 1970;57:349-52.

Price AJ, Jones NAG, Webb PJ, Chan PSF, Mistry FM, Kakkar VV. Do tourniquets prevent deep vein thrombosis? J Bone Joint Surg [Br] 1980;62-B:529.

Sagar S, Stamatakis JD, Thomas DP, Kakkar VV. Oral contraceptives, antithrombin-III activitiy, and postoperative deep-vein thrombosis. Lancet 1976;i:509-11.

Salvati ES, Lachiewicz P. Thromboembolism following total hip-replacement arthroplasty. J Bone Joint Surg [Am] 1976;58-A:921-5.

Salzman EW, Harris WH. Prevention of venous thromboembolism in orthopaedic patients. J Bone Joint Surg [Am] 1976;58-A:903-13.

Sevitt S. The structure and grovith of valve-pocket thrombi in femoral veins. J Clin Pathol 1974;27:517-28.

Sharp AA. Therapeutic use of fibrinolytic activators. In: Forbes CD, Mackay N, eds. The detection and treatment of thrombo-embolism. A symposium held at the Royal College of Physicians and Surgeons, Glasgow, April 1976. Ashtead, Surrey: The Trust for Education and Research in Therapeutics, 1977:141-6.

Shaw A. Physical methods of prophylaxis for deep vein thrombosis. In: Forbes CD, Mackay N, eds. The detection and treatment of thrombo-embolism. A symposium held at the Royal College of Physicians and Surgeons, Glasgow, April 1976. Ashtead, Surrey: The Trust for Education and Research in Therapeutics, 1977:137-40.

Singer J. Value of clinical signs in diagnosis of deep venous thrombosis. Lancet 1980;i:1186.

Stamatakis JD, Kakkar VV, Sagar S, Lawrence D, Nairn D, Bentley PG. Femoral vein thrombosis and total hip replacement. Br Med J 1977;ii:223-5.

Von Kaulla E, Droegemueller W, Aoki N, Von Kaulla KN. Antithrombin III depression and thrombin generation acceleration in women taking oral contraceptives. Am J Obstet Gynecol 1971;109:868-73.

Warlow C, Beattie AG, Terry G, Ogston D, Kenmure ACF, Douglas AS. A double-blind trial of low doses of subcutaneous heparin in the prevention of deep-vein thrombosis after myocardial infarction. Lancet 1973;ii:934-6.

Williams HT. Prevention of postoperative deep-vein thrombosis with perioperative subcutaneous heparin. Lancet 1971;ii:950-2.

Yin ET, Wessler S, Butler JV. Plasma heparin. A unique, practical, sub-microgram-sensitive assay. J Lab Clin Med 1973;81:298-310. 